\title{
Hierarchical Identity Based Encryption with Polynomially Many Levels
}

\author{
Craig Gentry ${ }^{1}$ and Shai Halevi ${ }^{2, \star}$ \\ ${ }^{1}$ Stanford \& IBM \\ ${ }^{2}$ IBM
}

\begin{abstract}
We present the first hierarchical identity based encryption (HIBE) system that has full security for more than a constant number of levels. In all prior HIBE systems in the literature, the security reductions suffered from exponential degradation in the depth of the hierarchy, so these systems were only proven fully secure for identity hierarchies of constant depth. (For deep hierarchies, previous work could only prove the weaker notion of selective-ID security.) In contrast, we offer a tight proof of security, regardless of the number of levels; hence our system is secure for polynomially many levels.

Our result can very roughly be viewed as an application of Boyen's framework for constructing HIBE systems from exponent-inversion IBE systems to a (dramatically souped-up) version of Gentry's IBE system, which has a tight reduction. In more detail, we first describe a generic transformation from "identity based broadcast encryption with key randomization" (KR-IBBE) to a HIBE, and then construct KR-IBBE by modifying a recent construction of IBBE of Gentry and Waters, which is itself an extension of Gentry's IBE system. Our hardness assumption is similar to that underlying Gentry's IBE system.
\end{abstract}

\section{Introduction}

Identity-Based Encryption (IBE) is a public-key encryption scheme where one's public key can be freely set to any value (such as one's identity): An authority that holds a master secret key can take any arbitrary identifier and extract a secret key corresponding to this identifier. Anyone can then encrypt messages using the identifier as a public encryption key, and only the holder of the corresponding secret key can decrypt these messages. This concept was introduced by Shamir 19, a partial solution was proposed by Maurer and Yacobi 18, and

The original version of this chapter was revised: The copyright line was incorrect. This has been corrected. The Erratum to this chapter is available at DOI: 10.1007/978-3-642-00457-5_36

* Research was sponsored by US Army Research laboratory and the UK Ministry of Defense and was accomplished under Agreement Number W911NF-06-3-0001. The views and conclusions contained in this document are those of the authors and should not be interpreted as representing the official policies, either expressed or implied, of the US Army Research Laboratory, the U.S. Government, the UK Ministry of Defense, or the UK Government. The US and UK Governments are authorized to reproduce and distribute reprints for Government purposes notwithstanding any copyright notation hereon.

O. Reingold (Ed.): TCC 2009, LNCS 5444, pp. 437-456. 2009.

(C) Springer-Verlag Berlin Heidelberg 2009 
the first fully functional IBE systems were described by Boneh and Franklin $[5]$ and Cocks [1].

IBE systems can greatly simplify the public-key infrastructure for encryption solutions, but they are still not as general as one would like. Many organizations have an hierarchical structure, perhaps with one central authority, several sub-authorities and sub-sub-authorities and many individual users, each belonging to a small part of the organization tree. We would like to have a solution where each authority can delegate keys to its sub-authorities, who in turn can keep delegating keys further down the hierarchy to the users. The depth of the hierarchy can range from two or three in small organizations, up to ten or more in large ones. An IBE system that allows delegation as above is called Hierarchical Identity-Based Encryption (HIBE). In HIBE, messages are encrypted for identity-vectors, representing nodes in the identity hierarchy. This concept was introduced by Horwitz and Lynn [17, who also described a partial solution to it, and the first fully functional HIBE system was described by Gentry and Silverberg [15.

The security model for IBE and HIBE systems postulates an attacker that can adaptively make "key-reveal" queries, thereby revealing the decryption keys of identities of its choice. The required security property asserts that such an attacker still cannot break the encryption at any identity other than those for which it issued key-reveal queries. (Or in the case of HIBE, other than those for which it issued key-reveal queries or their descendants.)

For the first IBE and HIBE systems, the only known proofs of security are carried out in the random-oracle model. Canetti et al. 9] introduced a weaker notion of security called selective-ID, where the attacker must choose the identity to attack before the system parameters are chosen (but can still make adaptive keyreveal queries afterward). They proved that a variant of the Gentry-Silverberg system is secure in this model even without random oracles. Boneh and Boyen described a more efficient selective-ID HIBE [1], and later described a fully secure IBE system without a random oracle [2. Waters 22] described what is currently the most practical adaptively-secure HIBE system without random oracles.

All currently known fully-secure HIBE systems, however, suffer from loose security reductions (whether they use random oracles or not). Specifically, they lose a multiplicative factor of $\Omega(q / \ell)^{\ell}$ in the success probability, where $q$ is the number of key-reveal queries and $\ell$ is the depth of the identity hierarchy. This means that asymptotically these reductions can only be used for hierarchies of constant depth. When considering concrete parameters, these reductions are only meaningful for hierarchies of depth two or three.

Gentry 13 proposed the first adaptively-secure IBE system without random oracles that has a tight reduction to its underlying hard problem. Recently Gentry and Waters extended Gentry's IBE to construct an adaptively-secure identity based broadcast encryption (IBBE) system without random oracles [16], whose security is tightly based on a related hard problem. Our HIBE system builds on the Gentry-Waters system. 
Boyen [8] proposed a framework for constructing HIBE systems from exponentinversion IBE systems. Specifically, Boyen described some properties of pairingbased IBE systems (called parallel IBE and linear IBE), and proved that an IBE system with these properties can be transformed to HIBE with comparable security. Boyen noted that Gentry's IBE does not quite fit within this template, and left it as an open problem to construct a HIBE system from Gentry's IBE system. Our system, which solves this problem, does not quite fit within Boyen's framework, yet our approach owes much to Boyen's idea.

We construct the first fully-secure HIBE with a tight proof of security. Namely, ours is the first HIBE system that can be proven fully secure for more than a small constant number of levels. This solves an open problem posed in 1512223138 . Similarly to the systems of Gentry [13] and Gentry-Waters [16], we exhibit a tight reduction, albeit to a problem whose instances are of size linear in $q+\ell$.

\section{$1.1 \quad$ Loose and Tight Reductions}

On a high level, the reason that most IBE systems have loose reductions is that those reductions involve the following trade-off: For each identity ID, either the simulator knows a decryption key for ID, or it doesn't. If it knows a key for ID then it does not learn anything new if the adversary chooses ID as the target identity to attack, since it could have used the decryption key to learn the same information. And if the simulator does not know a decryption key for ID then it must abort if the adversary makes a key-reveal query for this identity.

The crucial difference in the security proof of Gentry's IBE 13 is that there are many different decryption keys for each identity, and the simulator knows a small subset of these keys. Thus, the simulator can answer every key-reveal query without aborting, but still learn something when the adversary choses that identity for the challenge ciphertext. In this sense, Gentry's IBE system follows the universal hash proof paradigm of Cramer and Shoup [12: Given a wellformed ciphertext, all the decryption keys recover the same message, but they recover different messages when the ciphertext is mal-formed (in a certain sense). The adversary is assumed to have a non-negligible advantage when the challenge ciphertext is well-formed, but has essentially no advantage (statistically) when it is mal-formed; the adversary's different behavior in these cases allows the simulator to solve the underlying decision problem. Gentry's reduction uses an underlying hard problem that has a large problem instance (size $\theta(q)$ ), to ensure that the adversary cannot use its $q$ key-reveal queries to determine what keys the simulator possesses for the target identity. In this work we extend Gentry's IBE system and proof to the case of a HIBE.

\subsection{Constructing HIBE, Step 1: From IBBE to HIBE}

In our quest to construct HIBE, we use as an intermediate step a specific type of identity-based broadcast encryption (IBBE). An IBBE system can be seen as somewhere in between regular IBE and HIBE: It allows a sender to encrypt a message to a set identities, and each member of this set can use its own key 
to decrypt the message. This is somewhat similar to HIBE, in that encryption is targeted at a group of identities (similarly to the identity vector in HIBE) 1 However, IBBE is simpler than HIBE since decryption keys correspond only to single identities (see Section [2.2).

As a first step in constructing HIBE systems, we provide a generic transformation from IBBE to HIBE. This transformation, however, requires an "augmented IBBE system" that also has decryption keys corresponding to sets of identities (for decrypting ciphertexts that were encrypted for these sets). Specifically, we require a key-randomizable identity based broadcast encryption (KR-IBBE), where it is possible to generate a uniformly random decryption key $K_{S}$ for a set of identities $S$ from any decryption key $K_{S^{\prime}}$ for $S^{\prime} \subset S$ (see Section [2.3). $\mathrm{KR}$-IBBE is rather close to HIBE, but a major difference is that security is defined with respect to an adversary that can only ask for decryption keys corresponding to single identities, not for sets of identities. Hence it is still simpler to design KR-IBBE and use our transformation than to design a HIBE "from scratch."

\subsection{Constructing HIBE, Step 2: Constructing KR-IBBE}

Even with the simplification of KR-IBBE, our construction and its proof are still rather complex. Part of the reason for the complexity of our system and proof stems from the inherent tension between the key-randomization requirement and the Cramer-Shoup proof paradigm: On one hand, key-randomization implies in particular that one can generate a random decryption key for an identity set $S$ from any fixed valid encryption key for the same set. On the other hand, the Cramer-Shoup paradigm require that the simulator be able to generate only a small subset of the decryption keys for the target identity set.

Our proof resolves this tension by going through an intermediate step in which we replace the full-randomization requirement with "pseudo-randomization": Namely, from each fixed valid encryption key we can only generate a small subset of the decryption keys, but this small subset still looks random. In our case, the difference between "fully-random" and "pseudo-random" keys is that "fully random" keys are taken from some linear space and "pseudo-random" keys are taken from a proper subspace of this linear space. These being linear spaces of group elements, they are indistinguishable under the Decision Linear Assumption 4 .

We prove the security of the "pseudo-random" system using techniques and hard problems analogous to those used by Gentry and Waters in [16], but we we need to make rather substantial modifications to the system given in [16. Most notably, the randomization requirement seems to imply that we cannot have scalars in the decryption key, so we must convert everything into vectors of group elements.

\footnotetext{
${ }^{1}$ We use IBBE as a tool for constructing HIBE, so we consider a variant where the intended recipients must be enumerated explicitly by the encryption procedure. Note that it is more common for IBBE to have the "revoked" recipients enumerated on encryption. Arguably, our variant should have been called multicast encryption.
} 


\section{HIBE and IBBE: Definitions}

For simplicity, we define our encryption systems as key encapsulation mechanisms (KEM). The standard transformation from KEM to encryption is ignored here.

\subsection{Hierarchical Identity-Based Encryption}

A HIBE system consists of the following five procedures:

$\operatorname{Setup}(\lambda, \ell)$ Takes as input a security parameter $\lambda$ and the hierarchy depth $\ell$. It outputs a public key $P K$ and a master secret key $S K$. The public key implies also a key space $\mathcal{K}(P K)$ and an identity space $\mathcal{I D}(P K)$, and hierarchical identities are (ordered) tuples in $\mathcal{I D}(P K) \leq \ell$.

KeyGen $(P K, S K$, ID) Takes as input the public key $P K$ and master secret key $S K$, and an identity vector ID $=\left[\mathrm{ID}_{1}, \ldots, \mathrm{ID}_{t}\right] \in \mathcal{I D}(P K)^{\leq \ell}$. It outputs a decryption key $K_{\mathbf{I D}}$ for ID.

KeyDerive $\left(P K, \mathbf{I D}, K_{\mathbf{I D}}, \mathbf{I D}^{\prime}\right)$ Takes as input the public key $P K$, the identity vector ID and corresponding decryption key $K_{\mathbf{I D}}$, and another vector ID' such that ID is a prefix of ID'. It outputs a decryption key $K_{\mathbf{I D}^{\prime}}$ for $\mathbf{I D}^{\prime}$.

$\mathrm{KEM}(P K, \mathbf{I D})$ Takes as input the public key $P K$ and identity vector ID. It outputs a pair $(K, C)$, where $K$ is the KEM key (from the key space $\mathcal{K}(P K)$ ) and $C$ is the ciphertext.

$\operatorname{Decrypt}\left(P K, C, \mathbf{I D}, K_{\mathbf{I D}}\right)$ On input the public key $P K$, ciphertext $C$, identity vector ID and corresponding decryption key $K_{\mathbf{I D}}$. It outputs the corresponding KEM key $K$ (or an error message $\perp$ ).

We require the usual "completeness", namely that decryption with the correct decryption key always recovers the correct KEM key. In particular, setting $(P K, S K) \leftarrow \operatorname{Setup}(\lambda, \ell)$ and fixing any chain of identity vectors $\mathbf{I D}_{1}, \mathbf{I D}_{2}, \ldots, \mathbf{I D}_{t}$ with each $\mathbf{I D}_{i}$ a prefix of $\mathbf{I D}_{i+1}$, if we set $K_{\mathbf{I D}_{1}} \leftarrow \operatorname{KeyGen}\left(P K, S K, \mathbf{I D}_{1}\right)$ and then $K_{\mathbf{I D}_{i}} \leftarrow$ KeyDerive $\left(P K, \mathbf{I D}_{i-1}, K_{\mathbf{I D}_{i-1}}, \mathbf{I D}_{i}\right)$ for $i=2, \ldots, t$ and $(K, C) \leftarrow$ $\operatorname{KEM}\left(P K, \mathbf{I D}_{t}\right)$, then we have $\operatorname{Decrypt}\left(P K, C, \mathbf{I D}_{t}, K_{\mathbf{I D}_{t}}\right)=K$ (with probability one).

Security 1 Chosen-plaintext security for a HIBE system $\mathcal{E}$ against an adversary $A$ is defined by the following game between $A$ and a "challenger" (both given the parameters $\lambda, \ell$ as input):

Setup: The challenger runs $(P K, S K) \leftarrow \mathcal{E}$. Setup $(\lambda, \ell)$ and gives $P K$ to $A$.

Key-Reveal: The adversary $A$ makes adaptive key-reveal queries to the challenger, each consisting of an identity vector ID $=\left[\mathrm{ID}_{1}, \ldots, \mathrm{ID}_{t}\right] \in \mathcal{I D}(P K) \leq \ell$. If the adversary already made the challenge query and ID is a prefix of the target identity ID* then the challenger ignores this query, and otherwise it returns to the adversary the decryption key $K_{\mathbf{I D}} \leftarrow \mathcal{E}$. KeyGen $(P K, S K$, ID).

${ }^{2}$ Our security definition below ignores the delegation issue that was noted by Shi and Waters 20], see discussions later in this section. 
Challenge: The adversary queries the challenger with the target identity vector $\mathbf{I D}^{*}=\left[\mathrm{ID}_{1}^{*}, \ldots, \mathrm{ID}_{t}^{*}\right] \in \mathcal{I D}(P K)^{\leq \ell}$. If the adversary already made a challenge query before, or if it made a key-reveal query for any prefix of the target identity ID* then the challenger ignores this query. Otherwise the challenger sets $\left(K_{1}, C\right) \leftarrow \mathcal{E} \cdot \operatorname{KEM}\left(P K, \mathbf{I D}^{*}\right)$, chooses another random key $K_{0} \in_{R} \mathcal{K}(P K)$ and a "challenge bit" $\sigma \in_{R}\{0,1\}$, and returns $\left(K_{\sigma}, C\right)$ to the adversary.

The adversary can make many Key-Reveal queries and one Challenge query, in whatever order. Then it halts, outputting a guess $\sigma^{\prime}$ for the challenge bit $\sigma$. The HIBE advantage of $A$ is

$$
\operatorname{AdvHIBE}_{A}^{\mathcal{E}}(\lambda, \ell)=\operatorname{Pr}[A \Rightarrow 1 \mid \sigma=1]-\operatorname{Pr}[A \Rightarrow 1 \mid \sigma=0]
$$

Definition 1 (CPA-secure HIBE). The system $\mathcal{E}$ is CPA-secure if for any efficient adversary $\mathcal{A}$ and any $\ell=\operatorname{poly}(\lambda)$ it holds that $\operatorname{AdvHIBE}_{A}^{\mathcal{E}}(\lambda, \ell(\lambda))$ is negligible in $\lambda$.

CCA-security is defined similarly, where the adversary can also make decryption queries (except for decrypting the target ciphertext by the target identity vector).

Key delegation. Shi and Waters observed recently [20] that definitions such as the one above are incomplete model of the real world. In the definition above the adversary only sees decryption keys that were generated by KeyGen, whereas compromised nodes in the real world have keys that were generated by KeyDerive. This could be significant, since different delegation paths could result in different distributions of secret keys. Shi wand Waters presented a more elaborate definition in which the adversary is allowed to specify a delegation path and obtain a key that was generated using this delegation path.

For our construction, the key-randomization property ensures that the distribution of keys is nearly identical, whether they are generated by KeyGen or by KeyDerive. Hence, we only prove security with respect to this simplified definition.

\subsection{Identity-Based Broadcast Encryption}

An IBBE system consists of the procedures (Setup, KeyGen, KEM, Decrypt). Setup, KeyGen, and KEM are similar to HIBE, except that KeyGen can only be used for single identities (not identity vectors), and KEM gets a set of identities $S$ instead of an ordered vector. Decrypt is defined as follows:

$\operatorname{Decrypt}\left(P K, C, S, \mathrm{ID}, K_{\mathrm{ID}}\right)$ On input the public key $P K$, ciphertext $C$, identity set $S=\left\{\mathrm{ID}_{1}, \ldots, \mathrm{ID}_{t}\right\}$ (with $t \leq \ell$ ) and the decryption key $K_{\mathrm{ID}}$ for some ID $\in S$. It outputs the corresponding KEM key $K$ (or an error message $\perp$ ).

The security definition for IBBE is similar to the one for HIBE, the difference being that the adversary can only make key-reveal queries for single identities rather than identity-vectors. See the long version [14 for the formal definitions. 


\subsection{Key-Randomizable IBBE}

To construct HIBE systems, we will use "augmented IBBE systems" that also have decryption keys corresponding to sets of identities: A decryption key corresponding to an identity-set $S$ makes it possible to decrypt ciphertexts that were created with respect to this set. A Key-Randomizable Identity-Based Broadcast Encryption system (KR-IBBE) is an IBBE system with extended key generation KeyGen*, extended decryption Decrypt*, and key-derivation KeyDerive, as follows:

KeyGen* $(P K, S K, S)$ Takes as input the public key $P K$, master secret key $S K$, and an identity set $S=\left\{\mathrm{ID}_{1}, \ldots, \mathrm{ID}_{t}\right\} \in \mathcal{I D}(P K) \leq \ell$, and outputs a decryption key $K_{S}$ for $S$. We require that KeyGen* $(P K, S K, S)$ degenerates to the original KeyGen when $S$ is a singleton set $S=\{$ ID $\}$.

KeyDerive $\left(P K, S, K_{S}, S^{\prime}\right)$ Takes as input the public key $P K$, an identity set $S$ and corresponding decryption key $K_{S}$, and a superset $S^{\prime} \supseteq S$, and outputs a decryption key $K_{S^{\prime}}$ for $S^{\prime}$ 3

$\operatorname{Decrypt}^{*}\left(P K, C, S, K_{S}\right)$ Takes as input the public key $P K$, an identity set $S$, ciphertext $C$ that was generated with respect to $S$, and the decryption key $K_{S}$ for $S$. It outputs the KEM key $K$ (or an error message $\perp$ ).

We stress that we make no security requirements regarding these additional procedures: the CPA-security game is still defined with respect to the original four procedures Setup,KeyGen,KEM, Decrypt. However, we do make some functionality requirements, specifically the standard "completeness" requirement on Decrypt* and a distribution requirement on KeyDerive.

The "completeness" requirement says that for any $(P K, S K) \leftarrow \operatorname{Setup}(\lambda, \ell)$ and any set of identities $S$, if we set $K_{S} \leftarrow \operatorname{KeyGen}^{*}(P K, S K, S)$ and $(K, C) \leftarrow$ $\operatorname{KEM}(P K, S)$, then we get Decrypt ${ }^{*}\left(P K, C, S, K_{S}\right)=K$ (with probability one).

The distribution requirement says for any $(P K, S K) \leftarrow \operatorname{Setup}(\lambda, \ell)$, any two sets of identities $S \subseteq S^{\prime}$, and any decryption key $K_{S} \leftarrow \operatorname{KeyGen}^{*}(P K, S K, S)$, the output distributions of KeyGen ${ }^{*}\left(P K, S K, S^{\prime}\right)$ and KeyDerive $\left(P K, S, K_{S}, S^{\prime}\right)$ are almost identical. (That is, their statistical distance is negligible in $\lambda$.)

Remark. Due to the distribution requirement above, our transformation from key-randomizable IBBE to HIBE in Section 3 results in a system where the decryption keys generated by KeyDerive have the same distribution as the ones generated by KeyGen. As we pointed out before, this property allows us to ignore the delegation issue of Shi and Waters [20].

\section{From Key-Randomizable IBBE to HIBE}

The transformation from key-randomizable IBBE to HIBE is quite straightforward: we use collision-resistant hashing to map identity-vectors to identitysets, and then just use each of the procedures Setup, KeyGen*, KeyDerive, KEM,

\footnotetext{
${ }^{3}$ Note that in this setting of broadcast encryption, keys corresponding to smaller sets are "more powerful" than ones corresponding to larger sets: one can derive a key for the superset $S^{\prime}$ from any key for a subset $S$, but not the other way around.
} 
Decrypt* as-is. The only non-trivial aspect of this transformation is the security reduction, since the HIBE adversary can make key-reveal queries on identityvectors whereas the IBBE adversary can only ask for keys of "top level" single identities. We handle this difference by having the reduction algorithm generate decryption keys differently than is done in the system, which is where we need the distribution requirement of key randomization.

\subsection{The Transformation}

Let $\mathcal{E}=$ (Setup, KeyGen*, KeyDerive, KEM, Decrypt*) be a key-randomizable IBBE system, and we assume that we have a "matching" collision resistant hash function $H$ that can hash identity-vectors into the identity space of $\mathcal{E} \mathbb{W e}$ use $H$ to hash identity vectors in the HIBE system into identity sets for $\mathcal{E}$ by setting:

$$
\boldsymbol{H}\left(\mathrm{ID}_{1}, \ldots, \mathrm{ID}_{i}\right) \stackrel{\text { def }}{=}\left\{H\left(\mathrm{ID}_{1}\right), H\left(\mathrm{ID}_{1}, \mathrm{ID}_{2}\right), \ldots, H\left(\mathrm{ID}_{1}, \mathrm{ID}_{2}, \ldots, \mathrm{ID}_{i}\right)\right\}
$$

Note that short of finding collisions in $H$, we can only get $H\left(\mathrm{ID}_{1}, \mathrm{ID}_{2}, \ldots, \mathrm{ID}_{i}\right) \in$ $\boldsymbol{H}\left(\mathbf{I D}^{\prime}\right)$ if $\left(\mathrm{ID}_{1}, \mathrm{ID}_{2}, \ldots, \mathrm{ID}_{i}\right)$ is a prefix of ID'. Then we construct a HIBE system as follows 5

HIBE.Setup $(\lambda, \ell)$ : Set $\left(S K_{0}, P K_{0}\right) \leftarrow \mathcal{E} \cdot \operatorname{Setup}(\lambda, \ell)$. Output $S K$ and $P K$, which are the same as $S K_{0}$ and $P K_{0}$, except that each includes a description of the hash function $H$ as above.

HIBE.KeyGen $\left(P K, S K\right.$, ID): Set $S \leftarrow \boldsymbol{H}\left(\right.$ ID) (as above), $K_{S} \leftarrow \mathcal{E}$.KeyGen* $\left(P K_{0}\right.$, $\left.S K_{0}, S\right)$ and output $K_{\text {ID }}=K_{S}$.

HIBE.KeyDerive $\left(P K\right.$, ID, $\left.K_{\mathbf{I D}}, \mathbf{I D}^{\prime}\right)$ : Set $S \leftarrow \boldsymbol{H}($ ID $)$ and $S^{\prime} \leftarrow \boldsymbol{H}\left(\mathbf{I D}^{\prime}\right)$, and note that $S \subseteq S^{\prime}$ since ID is a prefix of ID $^{\prime}$. Also let $K_{S}=K_{\mathbf{I D}}$, compute $K_{S^{\prime}} \leftarrow$ $\mathcal{E}$.KeyDerive $\left(P K_{0}, S, K_{S}, S^{\prime}\right)$ and output $K_{\mathbf{I D}^{\prime}}=K_{S^{\prime}}$.

HIBE.KEM $(P K, S)$ : Set $S \leftarrow \boldsymbol{H}($ ID $)$, compute $(K, C) \leftarrow \mathcal{E} \cdot \operatorname{KEM}\left(P K_{0}, S\right)$ and output $(K, C)$.

HIBE.Decrypt $\left(P K, C, \mathbf{I D}, K_{\mathbf{I D}}\right):$ Set $S \leftarrow \boldsymbol{H}($ ID $)$ and $K_{S}=K_{\mathbf{I D}}$, and return $\mathcal{E}$.Decrypt ${ }^{*}\left(P K_{0}, C, S, K_{S}\right)$.

Theorem 1. Suppose that there exists a HIBE adversary $\mathcal{A}$ that breaks $C P A$ security (resp. CCA security) of the HIBE construction with advantage $\epsilon$. Then, there exists an IBBE adversary $\mathcal{B}$ and a collision finder $\mathcal{B}^{\prime}$, both running in about the same time as $\mathcal{A}$, such that $\mathcal{B}^{\prime}$ finds a hash function collision with some probability $\epsilon^{\prime}$ and $\mathcal{B}$ breaks the CPA security (resp. CCA security) of the underlying $K R$-IBBE system $\mathcal{E}$ with advantage $\epsilon-\epsilon^{\prime}$.

\footnotetext{
${ }^{4}$ The identity space in our IBBE system from Section $\left[5\right.$ is $\mathbb{Z}_{q}$ for a large $q$, so "matching" a hash function is easy.

${ }^{5}$ Note that this transformation is completely black box; in particular, it does not depend on whether or not the IBBE system uses a bilinear map.
} 
The proof is in the long version 14. The only non-trivial aspect of the proof is that to get a key for the set $S \leftarrow \boldsymbol{H}\left(\mathrm{ID}_{1}, \ldots, \mathrm{ID}_{t}\right)$, the simulator makes a query for the singleton key of the identity $\mathrm{ID}_{t}^{\prime}=H\left(\mathrm{ID}_{1}, \ldots, \mathrm{ID}_{t}\right) \in S$, and then uses key-derivation to get the key for $S$.

\section{Notations and Preliminaries}

We now introduce notations and hardness assumption that are used to establish our key-randomizable IBBE in Section 5. We denote the set of integers from $m$ to $n$ (inclusive) by $[m, n]$. We denote polynomials by uppercase letters in San-serif font, for example P, Q, T, etc. We use the following simple fact about polynomials:

Lemma 1. For any polynomial $\mathrm{P}(x)$ and any scalar $a, \mathrm{P}(x)-\mathrm{P}(a)$ is divisible by $x-a$. In other words, $\frac{\mathrm{P}(x)-\mathrm{P}(a)}{x-a}$ is a polynomial (without denominator) of degree $\operatorname{deg}(\mathrm{P})-1$.

\subsection{Bilinear Maps and Our Additive Notations}

Our system and its security proof make heavy use of linear algebra. We therefore use additive notations for all the groups that are involved in the system. Specifically, we use $\mathbb{Z}_{q}$ — the field of integers modulo a prime $q$ - as our base scalar field, and we have two order- $q$ groups that we call the source group $\mathbb{G}$ and target group $\mathbb{G}_{T}$, both of which can be viewed as vector spaces over $\mathbb{Z}_{q}$.

Throughout the writeup we denote elements of the source group with a hat over lowercase letters (e.g., $\hat{a}, \hat{b}$, etc.) and elements of the target group with a tilde $(\tilde{a}, \tilde{b}$, etc.). Scalars will be denoted with no decorations (e.g., $a, b$, and sometimes also $\tau, \rho$, etc.)

We will make use of an efficiently computable bilinear map from the source group to the target group e : $\mathbb{G} \times \mathbb{G} \rightarrow \mathbb{G}_{T}, 6$ such that for any two source-group elements $\hat{a}, \hat{b} \in \mathbb{G}$ and any two scalars $u, v \in \mathbb{Z}_{q}$ it holds that

$$
\mathrm{e}(u \cdot \hat{a}, v \cdot \hat{b})=u v \cdot \mathrm{e}(\hat{a}, \hat{b})
$$

The neutral elements in the groups $\mathbb{G}, \mathbb{G}_{T}$ are denoted by $\hat{0}, \tilde{0}$, respectively. We also denote by $\hat{1}$ some fixed generator in $\mathbb{G}$, which we consider to be part of the description of $\mathbb{G}$. We require that the mapping e is non-trivial, which means that $\mathrm{e}(\hat{1}, \hat{1})$ is a generator in $\mathbb{G}_{T}$, and we denote this generator by $\tilde{1}=\mathrm{e}(\hat{1}, \hat{1})$.

More generally, for a scalar $a \in \mathbb{Z}_{q}$, we denote the source-group element $a \cdot \hat{1}$ by $\hat{a}$, and the target-group element $a \cdot \tilde{1}=\mathrm{e}(\hat{a}, \hat{1})$ by $\tilde{a}$. Conversely, for an element $\hat{a} \in \mathbb{G}$, its discrete-logarithm based $\hat{1}$ is denoted $a \in \mathbb{Z}_{q}$. (Readers who are used to multiplicative notations may find it easier to think of $\hat{a}, \tilde{a}$ as denoting

\footnotetext{
${ }^{6}$ Our system can just as well use a-symmetric bilinear maps where you have two different source groups, e : $\mathbb{G}_{1} \times \mathbb{G}_{2} \rightarrow \mathbb{G}_{T}$. We chose to describe it for the symmetric case $\mathbb{G}_{1}=\mathbb{G}_{2}$ in order to avoid introducing even more notations.
} 
" $a$ in the exponent" in the appropriate groups.) Note also that in these notations, the discrete-logarithm of $\hat{a}$ with respect to $\hat{b}$ is just their "ratio" $\hat{a} / \hat{b}$, which is a scalar.

With these notations, we usually omit the map e altogether, and simply denote it as a "product" of two source-group elements:

$$
\hat{a} \cdot \hat{b} \stackrel{\text { def }}{=} \mathrm{e}(\hat{a}, \hat{b})=\tilde{a b} \in \mathbb{G}_{T}
$$

Note that the bi-linearity of e looks in these notations just like the natural commutative property of products $\hat{u a} \cdot \hat{v b}=u v \cdot \tilde{a b}$.

Below we slightly abuse notations to denote "powers of group elements": If $\hat{a}$ is a group element with discrete-logarithm $a$, then we denote $\hat{a}^{i} \stackrel{\text { def }}{=} a^{i} \cdot \hat{1}$ and we call $\hat{a}^{i}$ the $i$ 'th power of $\hat{a}$. 7

Vectors and Matrices. We extend our notations to vectors and matrices: A vector of scalars is denoted with no decoration $\boldsymbol{a}=\left[a_{1}, a_{2}, \ldots, a_{n}\right]$, a vector of source-group elements denoted with a hat, $\hat{\boldsymbol{a}}=\left[\hat{a}_{1}, \hat{a}_{2}, \ldots, \hat{a}_{n}\right]$, and a vector of target-group elements denoted with a tilde $\tilde{\boldsymbol{a}}=\left[\tilde{a}_{1}, \tilde{a}_{2}, \ldots, \tilde{a}_{n}\right]$. All these vectors are considered row vectors.

Matrices are denoted by uppercase letters, e.g., $A$ for a matrix of scalars, $\hat{A}$ for a matrix of source-group elements, and $\tilde{A}$ for a matrix of target-group elements. We denote the $i$ 'th row of $A$ by $A_{i}$, the sub-matrix consisting of rows $i, j, k$ by $A_{i, j, k}$, and the sub-matrix consisting of rows $i$ through $j$ is denoted $A_{i . . j}$. As usual, the transposed matrix of $A$ is denoted $A^{t}$.

We denote by $\operatorname{span}(\boldsymbol{x}, \boldsymbol{y}, \boldsymbol{z})$ the linear space that is spanned by the vectors $\boldsymbol{x}, \boldsymbol{y}, \boldsymbol{z}$, and also use the same notation to denote the uniform distribution over this space. For example, we use $\hat{\boldsymbol{u}} \leftarrow \hat{\boldsymbol{w}}+\operatorname{span}\left(\hat{A}_{1,2,4}\right)$ as a shorthand for the process of choosing three random scalars $a, b, c \in \in_{R} \mathbb{Z}_{p}$ and setting $\hat{\boldsymbol{u}} \leftarrow \hat{\boldsymbol{w}}+$ $a \hat{A}_{1}+b \hat{A}_{2}+c \hat{A}_{4}$.

Inner and Outer-Products. For vectors $\boldsymbol{a}, \boldsymbol{b}$, we denote their inner product by $\langle\boldsymbol{a}, \boldsymbol{b}\rangle \stackrel{\text { def }}{=} \sum_{i} a_{i} b_{i}$. We use the same inner-product notations also for vectors of source-group elements, namely:

$$
\langle\boldsymbol{a}, \hat{\boldsymbol{b}}\rangle=\langle\hat{\boldsymbol{b}}, \boldsymbol{a}\rangle \stackrel{\text { def }}{=} \sum_{i} a_{i} \hat{b}_{i}=\langle\boldsymbol{a}, \boldsymbol{b}\rangle \cdot \hat{1}, \text { and }\langle\hat{\boldsymbol{a}}, \hat{\boldsymbol{b}}\rangle \stackrel{\text { def }}{=} \sum_{i} \mathrm{e}\left(\hat{a}_{i}, \hat{b}_{i}\right)=\langle\boldsymbol{a}, \boldsymbol{b}\rangle \cdot \tilde{1}
$$

It is easy to check that all the commutative, associative, and distributive properties of inner products hold for both scalars and group elements.

Similar notations apply to matrix multiplication, for either scalar matrices or group-element matrices. For example, if $A$ is an $\ell \times m$ scalar matrix and $\hat{B}$ is an $m \times n$ matrix of source-group elements, then $A \hat{B} \in \mathbb{G}[\ell \times n]$ is a matrix of source-group elements whose $i, j$ element is the inner product of the $i$ 'th row

\footnotetext{
7 This abuse of notation may take some getting used to: notice that the $a$ 's themselves should be thought of as being "in the exponent." In multiplicative notation, this power of $\hat{a}$ would be denoted as something like $g^{\left(a^{i}\right)}$.
} 
of $A$ by the $j$ 'th column of $\hat{B}$. We also use $\boldsymbol{a} \times \boldsymbol{b}$ to denote the outer product of two vectors. Namely, the outer product of the $m$-vector $\boldsymbol{a}$ by the $n$-vector $\boldsymbol{b}$ is the $m \times n$ matrix obtained as the matrix product of the $m \times 1$ matrix $\boldsymbol{a}^{t}$ by the $1 \times n$ matrix $\boldsymbol{b}$. The same notation applies to vectors of group elements.

Linear Algebra. All the standard concepts from linear algebra behave just the same with either scalars or group elements. For example, if $\hat{A} \in \mathbb{G}[n \times n]$ is a square matrix of source-group elements and $A$ is the matrix of the discrete logarithm of all the elements in $\hat{A}$ (with respect to the fixed generator $\hat{1}$ ), then the inverse of $\hat{A}$ is $\hat{A}^{-1}=A^{-1} \cdot \hat{1}$. (Equivalently, the inverse of $\hat{A}$ is the unique matrix $\hat{B}$ such that $\hat{A} \cdot \hat{B}=\tilde{I}$.) Similarly, the rank of a scalar matrix is defined as usual, and the rank of a matrix of group elements is defined as the rank of their discrete-logarithm matrix.

\subsection{The BDHE-Set Assumption}

The BDHE-Set assumption (used also in [16]) is a parameterized generalization of the $t$-BDHI problem from 19 Recall that a $t$-BDHI adversary is given $t+1$ powers of a random source-group element, $\hat{1}, \hat{a}, \hat{a}^{2}, \ldots \hat{a}^{t}$, and it needs to distinguish the target-group element $\tilde{a}^{-1}$ from random.

An instance of the BDHE-Set assumption is parameterized by a set of integers $\mathcal{S} \subset \mathbb{Z}$ and another "target integer" $m$. The BDHE-Set adversary is given some powers of a random source-group element, $\left\{\hat{a}^{i}: i \in \mathcal{S}\right\}$, and it (roughly) needs to distinguish the target-group element $\tilde{a}^{m}$ from random. Denoting $\mathcal{S}+{ }_{q} \mathcal{S} \stackrel{\text { def }}{=}$ $\{i+j \bmod \lambda(q): i, j \in \mathcal{S}\}$, where $\lambda(q)$ is the order of elements modulo $q$, it is easy to see that if $\mathbb{G}$ is an order- $q$ bilinear-map group and $m \in \mathcal{S}+{ }_{q} \mathcal{S}$ then the problem is easy: Just choose some $i, j \in \mathcal{S}$ such that $i+j=m \bmod \lambda(q)$ and compute the bilinear map

$$
\mathrm{e}\left(\hat{a}^{i}, \hat{a}^{j}\right)=\hat{a}^{i} \cdot \hat{a}^{j}=\tilde{a}^{i+j}=\tilde{a}^{m}
$$

However, when $m \notin \mathcal{S}+_{q} \mathcal{S}$ then there does not seem to be an easy way of distinguishing $\tilde{a}^{m}$ from random given the source-group elements $\left\{\hat{a}^{i}: i \in \mathcal{S}\right\}$. The formal BDHE-Set assumption below is somewhat stronger, however, giving the adversary not the target group element $\tilde{a}^{m}$ itself, but rather two random source group elements whose product is $\tilde{a}^{m}$. Even so, this may be a reasonable assumption to make.

Definition 2 (Decision BDHE-Set). Fix a prime number $q$, a set of integers $\mathcal{S}$ and another integer $m \notin \mathcal{S}+{ }_{q} \mathcal{S}$. Also fix two order-q groups $\mathbb{G}$ and $\mathbb{G}_{T}$, admitting a non-trivial, efficiently computable bilinear map e: $\mathbb{G} \times \mathbb{G} \rightarrow \mathbb{G}_{T}$.

The $(\mathcal{S}, m)$-BDHE-Set problem with respect to $\mathbb{G}$ and $\mathbb{G}_{T}$ consists of the following experiment: Choose at random a scalar $a \in_{R} \mathbb{Z}_{q}^{*}$ and a bit $\sigma \in_{R}\{0,1\}$. If $\sigma=0$ then choose two random scalars $z_{1}, z_{2} \in_{R} \mathbb{Z}_{q}^{*}$, and if $\sigma=1$ then choose a random scalar $z_{1} \in_{R} \mathbb{Z}_{q}^{*}$ and set $z_{2} \leftarrow a / z_{1} \bmod q$. The BDHE-Set adversary

${ }^{8}$ This assumption is called $q$-BDHI in 11, but we use the letter $q$ as our group order. 
gets as input $\hat{a}^{i}=a^{i} \cdot \hat{1}$ for all $i \in \mathcal{S}$ and also $\hat{z}_{1}, \hat{z}_{2}$, and its goal is to guess the bit $\sigma$. The advantage of an adversary $A$ is defined as

$$
\begin{array}{r}
\operatorname{AdvBDHE}_{A}^{\mathcal{S}, m}\left(\mathbb{G}, \mathbb{G}_{T}\right) \stackrel{\text { def }}{=} \operatorname{Pr}\left[a, z_{1} \in \in_{R} \mathbb{Z}_{q}^{*}, z_{2} \leftarrow \frac{a}{z_{1}}, A\left(\left\{\hat{a}^{i}: i \in \mathcal{S}\right\}, \hat{z}_{1}, \hat{z}_{2}\right) \Rightarrow 1\right] \\
-\operatorname{Pr}\left[a, z_{1}, z_{2} \in R \mathbb{Z}_{q}^{*}, A\left(\left\{\hat{a}^{i}: i \in \mathcal{S}\right\}, \hat{z}_{1}, \hat{z}_{2}\right) \Rightarrow 1\right]
\end{array}
$$

Informally, the asymptotic Decision BDHE-Set assumption states that for any $m \notin \mathcal{S}+\mathcal{S}$ and a large enough prime $q$, efficient adversaries (that work in time $\operatorname{poly}(|\mathcal{S}|, \log q)$ only have insignificant advantage in the experiment from above. Making this formal is rather straightforward (though getting the quantification right takes some care).

Jumping ahead, for our system we use the assumption above with the target integer $m=-1$ and the set $\mathcal{S}$ defined as:

$$
\begin{aligned}
& \mathcal{S}= {[-2 h-2 \ell,-2 h-\ell-2] \cup[-h-\ell,-\ell-1] } \\
& \cup[0, \ell-1] \cup[h+\ell, 2 h+\ell] \cup[2 h+2 \ell, 3 h+2 \ell+1]
\end{aligned}
$$

where $\ell$ is the depth of the identity-hierarchy of the system and $h>\ell$ is some other parameter. (Specifically, if $q^{*}$ is a bound on the number of queries then $h=q^{*}+\ell+2$.) It is easy to check that indeed $m=-1 \notin \mathcal{S}+\mathcal{S}$.

The Linear Assumption. The decision linear assumption, first defined in 4], states (in our additive notations) that given the six source group elements $\hat{a}, \hat{b}, \hat{c}, \hat{d}, \hat{e}, \hat{f}$, it is hard to distinguish the case where these elements are completely random from the case where they are chosen at random subject to the condition $\hat{f} / \hat{c}=\hat{e} / \hat{b}+\hat{d} / \hat{a}$. (I.e., the discrete logarithm of $f$ relative to $c$ is the sum of the discrete logarithm of $e$ relative to $b$ and the discrete logarithm of $d$ relative to a.) Note that this assumption is equivalent to saying that given the matrix of group elements

$$
M=\left(\begin{array}{lll}
\hat{a} & \hat{0} & \hat{c} \\
\hat{0} & \hat{b} & \hat{c} \\
\hat{d} & \hat{e} & \hat{f}
\end{array}\right)
$$

it is hard to decide if this matrix is invertible or has rank two. In this work we use a slightly weaker variant of this assumption: Specifically, we assume that given a $3 \times 3$ matrix of source-group elements, it is hard to distinguish the case where this is a random invertible matrix from the case where it is a random rank-two matrix. (The advantage of an adversary in distinguishing these cases is

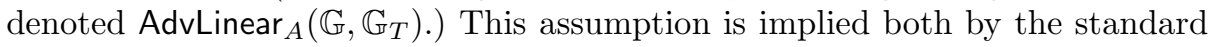
linear assumption from 4 and by our BDHE-Set assumption, but we make it a separate assumption just to make the exposition of our security-proof easier.

\section{A Key-Randomizable IBBE System}

Our system operates in prime-order bilinear-map groups. In the description below we assume that these order- $q$ groups are fixed "once and for all" and 
everyone knows their description. (An alternative description will include the group-generation as part of the Setup procedure.) We also fix the hierarchydepth of the system to some integer $\ell$.

The identity space of the system is the scalar field $\mathbb{Z}_{q}$, except that we have $\ell$ "forbidden identities" within this range: $\ell-1$ of them are arbitrary (and we set them to be $0,1, \ldots, \ell-2)$, and the last one is a random scalar $a$ that is chosen during Setup (see below).

Setup: Choose three random scalars $a, b, s \in Z_{q}$ and a random invertible matrix $A \in G[7 \times 7]$, and set $\hat{B}=\left(\hat{A}^{-1}\right)^{t}$. We note that the system only uses the top four rows of $\hat{A}$ and five rows of $\hat{B}$. The seventh dimension is only used in the security proof. Below we denote by $\boldsymbol{a}_{i}$ the vector $\boldsymbol{a}_{i} \stackrel{\text { def }}{=}\left[\begin{array}{lllll}1 & a & a^{2} & \ldots & a^{i}\end{array}\right]$.

- The master secret key is $S K=\left(\hat{B}_{1 . .6}, s, \boldsymbol{a}_{\ell}\right)$.

- The public key consists of three parts, $P K=\left(P K_{1}, P K_{2}, P K_{3}\right)$ with $P K_{1}$ consisting of a target-group element that is used to compute the KEM key, $P K_{2}$ consisting of multiples of the rows of $\hat{A}$ that are used to compute the ciphertext, and $P K_{3}$ consisting of multiples of the rows of $\hat{B}$ that are used only for key randomization. Specifically we have $P K_{1}=a^{\ell-1} \tilde{s}$ and

$$
\begin{aligned}
P K_{2}= & \{\underbrace{\left\{a^{i} \hat{A}_{1}: i=0, \ldots, \ell\right\}}_{\boldsymbol{a}_{\ell} \times \hat{A}_{1}}, s \hat{A}_{2}, \underbrace{\left\{a^{i} \hat{A}_{3}: i=0, \ldots, \ell-1\right\}}_{\boldsymbol{a}_{\ell-1} \times \hat{A}_{3}}, \hat{A}_{4}\} \\
P K_{3}= & \left\{\begin{array}{l}
b s \hat{B}_{1}, \quad a b s \hat{B}_{1}, \quad \hat{B}_{5}, \quad \hat{B}_{6},, \underbrace{\left\{a^{i} b \hat{B}_{1}: i=0, \ldots, \ell\right\}}_{b\left(\boldsymbol{a}_{\ell} \times \hat{B}_{1}\right)}, \\
\underbrace{\left\{a^{i} b \hat{B}_{2}: i=0, \ldots, \ell\right\}}_{b\left(\boldsymbol{a}_{\ell} \times \hat{B}_{2}\right)}, \underbrace{\left\{a^{i} b \hat{B}_{3}: i=0, \ldots, \ell+1\right\}}_{b\left(\boldsymbol{a}_{\ell+1} \times \hat{B}_{3}\right)}\}
\end{array}\right.
\end{aligned}
$$

$\operatorname{KeyGen}(P K, S K$, ID): Choose a key of $3 \ell-3$ seven-dimensional vectors of source-group elements as follows: Pick at random $r_{\mathrm{ID}} \in Z_{q}$ and set $\hat{K}_{\mathrm{ID}}=$ $\left(\hat{\boldsymbol{u}}_{\mathrm{ID}}, \hat{V}_{\mathrm{ID}}, \hat{W}_{\mathrm{ID}}, \hat{X}_{\mathrm{ID}}, \hat{\boldsymbol{y}}_{\mathrm{ID}}\right)$, where

$$
\begin{array}{ll}
\hat{\boldsymbol{u}}_{\mathrm{ID}}=\frac{s-r_{\mathrm{ID}}}{a-\mathrm{ID}} \hat{B}_{1} & \hat{V}_{\mathrm{ID}}=r_{\mathrm{ID}}\left(\boldsymbol{a}_{\ell-2} \times \hat{B}_{1}\right)\left(=\left\{r_{\mathrm{ID}} a^{i} \hat{B}_{1}: i=0, \ldots, \ell-2\right\}\right) \\
\hat{W}_{\mathrm{ID}}=\boldsymbol{a}_{\ell-2} \times \hat{B}_{2} \quad\left(=\left\{a^{i} \hat{B}_{2}: i=0, \ldots, \ell-2\right\}\right) \\
\hat{\boldsymbol{y}}_{\mathrm{ID}}=r_{\mathrm{ID}} a^{\ell-1} \hat{B}_{3}+\operatorname{span}\left(\hat{B}_{5,6}\right) \hat{X}_{\mathrm{ID}}=r_{\mathrm{ID}}\left(\boldsymbol{a}_{\ell-2} \times \hat{B}_{3}\right)\left(=\left\{r_{\mathrm{ID}} a^{i} \hat{B}_{3}: i=0, \ldots, \ell-2\right\}\right)
\end{array}
$$

Note that the $\hat{W}_{\text {ID }}$ component is the same for all identities (so it really belongs in the public key). It is included in the secret key only for the purpose of the key-randomization procedure below.

$\operatorname{KEM}(P K, S)$ : If $|S|<\ell$ then add to $S$ the first $\ell-|S|$ of the "forbidden identities" $0,1, \ldots$. Denote the resulting $\ell$ identities by $\left\{\mathrm{ID}_{1}, \mathrm{ID}_{2}, \ldots, \mathrm{ID}_{\ell}\right\}$. 
- Set the monic degree- $\ell$ polynomial $\mathrm{P}(x) \stackrel{\text { def }}{=} \prod_{i=1}^{\ell}\left(x-\mathrm{ID}_{i}\right)$, let $p_{0}, \ldots, p_{\ell}$ be the coefficients of $\mathrm{P}$ and denote $\boldsymbol{p} \stackrel{\text { def }}{=}\left[\begin{array}{lll}p_{0} & \ldots & p_{\ell}\end{array}\right]\left(\right.$ so $\left.\mathrm{P}(a)=\left\langle\boldsymbol{p}, \boldsymbol{a}_{\ell}\right\rangle\right)$.

- Choose at random $f_{0}, \ldots, f_{\ell-1} \in Z_{q}$ and denote $\boldsymbol{f} \stackrel{\text { def }}{=}\left[f_{0} f_{1} \ldots f_{\ell-1}\right]$ and $\mathrm{F}(x) \stackrel{\text { def }}{=} \sum_{i=0}^{\ell-1} f_{i} x^{i}$. Make sure that $\mathrm{F}\left(\operatorname{ID}_{i}\right) \neq 0$ for all $i=1, \ldots, \ell$ (otherwise re-choose $\mathrm{F}$ until this condition holds).

- Choose a random scalar $t \in Z_{q}$.

- Output the ciphertext containing the polynomial $F$ and the vector

$$
\hat{\boldsymbol{c}}=t(\underbrace{\mathrm{P}(a) \hat{A}_{1}}_{\boldsymbol{p}\left(\boldsymbol{a}_{\ell} \times \hat{A}_{1}\right)}+s \hat{A}_{2}+\underbrace{\mathrm{F}(a) \hat{A}_{3}}_{\boldsymbol{f}\left(\boldsymbol{a}_{\ell-1} \times \hat{A}_{3}\right)})+\operatorname{span}\left(\hat{A}_{4}\right)
$$

The implied KEM key is the target-group element $\tilde{k}=t \cdot P K_{1}=a^{\ell-1} t \tilde{s}$.

Remark. Note that the ciphertext include seven source group elements and $\ell$ scalars (to specify F). The ciphertext size can be reduced in a particular way, so that when encrypting to a set $S$ of size $m<\ell$ we only have $m$ scalars in the ciphertext: Instead of choosing F completely at random, we impose the condition that $\mathrm{F}(I D)=1$ for each of the "forbidden identities" that were added to $S$. This way, the encryptor can specify $\mathrm{F}$ using only the $m$ scalars $\mathrm{F}\left(\mathrm{ID}_{i}\right)$ for all $\mathrm{ID}_{i} \in S$. This optimization requires a small change to the proof of security, see remark at the end of Section 6. We also note that we can get a constant-size ciphertext by moving to the random-oracle model: the encryptor just sends some nonce, and $\mathrm{F}$ is determined by applying the random oracle to this nonce.

$\operatorname{Decrypt}\left(P K,(\mathrm{~F}, \hat{\boldsymbol{c}}), S, \mathrm{ID}, \hat{K}_{\mathrm{ID}}\right)$, where ID $\in S$. If $|S|<\ell$ then add to $S$ the first $\ell-|S|$ of the "forbidden identities" $0,1, \ldots$ Denote the resulting $\ell$ identities by $\left\{\mathrm{ID}_{1}, \mathrm{ID}_{2}, \ldots, \mathrm{ID}_{\ell}\right\}$. Parse the key as $\hat{K}_{\mathrm{ID}}=\left(\hat{\boldsymbol{u}}_{\mathrm{ID}}, \hat{V}_{\mathrm{ID}}, \hat{W}_{\mathrm{ID}}, \hat{X}_{\mathrm{ID}}, \hat{\boldsymbol{y}}_{\mathrm{ID}}\right)$, recalculate the monic $\ell$-degree polynomial $\mathrm{P}(x)=\prod_{i=1}^{\ell}\left(x-\mathrm{ID}_{i}\right)$, and do the following:

- Set $\mathrm{Q}_{\mathrm{ID}}(x) \stackrel{\text { def }}{=} \frac{\mathrm{P}(x)}{x-\mathrm{ID}}$ and $\mathrm{Q}^{\prime}{ }_{\mathrm{ID}}(x)=\mathrm{Q}_{\mathrm{ID}}(x)-a^{\ell-1}$. (That is, $\mathrm{Q}^{\prime}$ is the polynomial $Q$ without the top coefficient of $1 \cdot x^{\ell-1}$.) Denote the coefficient vector of $\mathrm{Q}_{\mathrm{ID}}^{\prime}$ by $\boldsymbol{q}_{\mathrm{ID}}^{\prime}=\left[\begin{array}{llll}q_{0} & q_{1} & \ldots & q_{\ell-2}\end{array}\right]$.

- Set $\mathrm{G}_{\mathrm{ID}}(x) \stackrel{\text { def }}{=} \frac{\mathrm{F}(x)-\mathrm{F}(\mathrm{ID})}{x-\mathrm{ID}}$ and denote the coefficient vector of $\mathrm{G}_{\mathrm{ID}}$ by $\boldsymbol{g}_{\mathrm{ID}}=$ $\left[\begin{array}{llll}g_{0} & g_{1} & \ldots & g_{\ell-2}\end{array}\right]$.

- Set

$$
\hat{\boldsymbol{d}}_{\mathrm{ID}}=\hat{\boldsymbol{u}}_{\mathrm{ID}}-\boldsymbol{q}_{\mathrm{ID}}^{\prime} \cdot \hat{W}_{\mathrm{ID}}-\frac{\boldsymbol{g}_{\mathrm{ID}} \cdot \hat{V}_{\mathrm{ID}}-\boldsymbol{q}_{\mathrm{ID}}^{\prime} \cdot \hat{X}_{\mathrm{ID}}-\hat{\boldsymbol{y}}_{\mathrm{ID}}}{\mathrm{F}(\mathrm{ID})}
$$

Finally, recover the KEM key as $\tilde{k}=\left\langle\hat{\boldsymbol{c}}, \hat{\boldsymbol{d}_{\mathrm{ID}}}\right\rangle$. 


\subsection{Correctness}

To argue correctness, we can rewrite

$$
\begin{aligned}
& \hat{\boldsymbol{d}}_{\mathrm{ID}}= \overbrace{\hat{\boldsymbol{u}}_{\mathrm{ID}}}^{\frac{s-r_{\mathrm{ID}}}{a-1 \mathrm{D}} \hat{B}_{1}}-\boldsymbol{q}_{\mathrm{ID}}^{\prime} \cdot \overbrace{\hat{W}_{\mathrm{ID}}}^{\boldsymbol{a}_{\ell-2} \times \hat{B}_{2}}-\frac{\boldsymbol{g}_{\mathrm{ID}} \cdot \overbrace{\hat{V}_{\mathrm{ID}}}^{r_{\mathrm{ID}} \boldsymbol{a}_{\ell-2} \times \hat{B}_{1}}-\boldsymbol{q}_{\mathrm{ID}}^{\prime} \cdot \overbrace{\hat{X}_{\mathrm{ID}}}^{r_{\mathrm{ID}} \boldsymbol{a}_{\ell-2} \times \hat{B}_{3}}-\overbrace{\hat{\boldsymbol{y}}_{\mathrm{ID}}}^{r_{\mathrm{ID}} a^{\ell-1} \hat{B}_{3}+\operatorname{span}\left(\hat{B}_{5,6}\right)}}{=} \\
&=\frac{s-r_{\mathrm{ID}}}{a-\mathrm{ID}} \hat{B}_{1}-\left\langle\boldsymbol{q}_{\mathrm{ID}}^{\prime}, \boldsymbol{a}_{\ell-2}\right\rangle \hat{B}_{2} \\
&-\frac{r_{\mathrm{ID}}}{\mathrm{F}(\mathrm{ID})}\left(\left\langle\boldsymbol{g}_{\mathrm{ID}}, \boldsymbol{a}_{\ell-2}\right\rangle \hat{B}_{1}-\left(\left\langle\boldsymbol{q}_{\mathrm{ID}}^{\prime}, \boldsymbol{a}_{\ell-2}\right\rangle+a^{\ell-1}\right) \hat{B}_{3}-\operatorname{span}\left(\hat{B}_{5,6}\right)\right) \\
&=\left(\frac{s-r_{\mathrm{ID}}}{a-\mathrm{ID}}-\frac{r_{\mathrm{ID}} \mathrm{G}_{\mathrm{ID}}(a)}{\mathrm{F}(\mathrm{ID})}\right) \hat{B}_{1}-\left(\mathrm{Q}_{\mathrm{ID}}(a)-a^{\ell-1}\right) \hat{B}_{2}+\frac{r_{\mathrm{ID}}}{\mathrm{F}(\mathrm{ID})}\left(\mathrm{Q}_{\mathrm{ID}}(a) \hat{B}_{3}+\operatorname{span}\left(\hat{B}_{5,6}\right)\right)
\end{aligned}
$$

Further developing the coefficient of $\hat{B}_{1}$ (using $\left.\mathrm{G}_{\mathrm{ID}}(a)(a-\mathrm{ID})=\mathrm{F}(a)-\mathrm{F}(\mathrm{ID})\right)$, we get

$$
\left(\frac{s-r_{\mathrm{ID}}}{a-\mathrm{ID}}-\frac{r_{\mathrm{ID}} \mathrm{G}_{\mathrm{ID}}(a)}{\mathrm{F}(\mathrm{ID})}\right)=\frac{\mathrm{F}(\mathrm{ID})\left(s-r_{\mathrm{ID}}\right)-r_{\mathrm{ID}} \mathrm{G}_{\mathrm{ID}}(a)(a-\mathrm{ID})}{\mathrm{F}(\mathrm{ID})(a-\mathrm{ID})}=\frac{s \cdot \mathrm{F}(\mathrm{ID})-r_{\mathrm{ID}} \cdot \mathrm{F}(a)}{\mathrm{F}(\mathrm{ID})(a-\mathrm{ID})}
$$

Examining the inner-product of $\hat{\boldsymbol{c}}$ with $\hat{\boldsymbol{d}}_{\mathrm{ID}}$, we use the fact that $\left\langle\hat{A}_{i}, \hat{B}_{j}\right\rangle$ is either 0 (when $i \neq j$ ) or $\tilde{1}$ (when $i=j$ ). Hence the span's of $\hat{A}_{4}$ and of $\hat{B}_{5,6}$ drop out completely, and we are left with the product of the matching coefficients only:

$$
\left\langle\hat{\boldsymbol{c}}, \hat{\boldsymbol{d}}_{\mathrm{ID}}\right\rangle=(\underbrace{t \mathrm{P}(a) \frac{s \cdot \mathrm{F}(\mathrm{ID})-r_{\mathrm{ID}} \cdot \mathrm{F}(a)}{\mathrm{F}(\mathrm{ID})(a-\mathrm{ID})}}_{\text {coefficients of } \hat{A}_{1}, \hat{B}_{1}}-\underbrace{t s\left(\mathrm{Q}_{\mathrm{ID}}(a)-a^{\ell-1}\right)}_{\text {coefficients of } \hat{A}_{2}, \hat{B}_{2}}+\underbrace{t \mathrm{~F}(a) \frac{r_{\mathrm{ID}}}{\mathrm{F}(\mathrm{ID})} \mathrm{Q}_{\mathrm{ID}}(a)}_{\text {coefficients of } \hat{A}_{3}, \hat{B}_{3}}) \cdot \tilde{1}
$$

The first term in the parenthesis can be simplified using $\mathrm{Q}_{\mathrm{ID}}(a)=\mathrm{P}(a) /(a-\mathrm{ID})$, so we get

$$
\begin{aligned}
\left\langle\hat{\boldsymbol{c}}, \hat{\boldsymbol{d}}_{\mathrm{ID}}\right\rangle & =t\left(\mathrm{Q}_{\mathrm{ID}}(a) \frac{s \cdot \mathrm{F}(\mathrm{ID})-r_{\mathrm{ID}} \cdot \mathrm{F}(a)}{\mathrm{F}(\mathrm{ID})}-s\left(\mathrm{Q}_{\mathrm{ID}}(a)-a^{\ell-1}\right)+\mathrm{F}(a) \frac{r_{\mathrm{ID}}}{\mathrm{F}(\mathrm{ID})} \mathrm{Q}_{\mathrm{ID}}(a)\right) \cdot \tilde{1} \\
& =t\left(\mathrm{Q}_{\mathrm{ID}}(a) s-\frac{r_{\mathrm{ID}} \mathrm{Q}_{\mathrm{ID}}(a) \mathrm{F}(a)}{\mathrm{F}(\mathrm{ID})}-\mathrm{Q}_{\mathrm{ID}}(a) s+a^{\ell-1} s+\frac{r_{\mathrm{ID}} \mathrm{Q}_{\mathrm{ID}}(a) \mathrm{F}(a)}{\mathrm{F}(\mathrm{ID})}\right) \cdot \tilde{1} \\
& =t \cdot a^{\ell-1} s \cdot \tilde{1}=\tilde{k}
\end{aligned}
$$

\subsection{Key Randomization}

Our key-randomization follows Boyen's idea from [8], where the key for identityset $S=\left\{\mathrm{ID}_{1}, \ldots, \mathrm{ID}_{m}\right\}$ consists of $m$ "shifted versions" of the keys, $r_{\mathrm{ID}_{1}}^{\prime} \hat{K}_{\mathrm{ID}_{1}}$, $\ldots, r_{\mathrm{ID}_{n}}^{\prime} \hat{K}_{\mathrm{ID}_{m}}$, such that $\sum_{i} r_{\mathrm{ID}_{i}}^{\prime}=1(\bmod q)$. Namely, the augmented procedure 
KeyGen* $(P K, S K, S)$ uses the same KeyGen procedure from above $m$ times to get $\hat{K}_{\mathrm{ID}_{i}} \leftarrow \operatorname{KeyGen}\left(P K, S K, \mathrm{ID}_{i}\right)$. Then for $i=1 \ldots m$ it chooses $r_{\mathrm{ID}_{i}}^{\prime} \in Z_{q}$ at random subject to the constraint $\sum_{i} r_{\mathrm{ID}_{i}}^{\prime}=1(\bmod q)$, and outputs the secret key

$$
\hat{K}_{S}=\left[r_{\mathrm{ID}_{1}}^{\prime} \hat{K}_{\mathrm{ID}_{1}}, \ldots, r_{\mathrm{ID}_{m}}^{\prime} \hat{K}_{\mathrm{ID}_{m}}\right]
$$

where $r_{\mathrm{ID}_{i}}^{\prime} \hat{K}_{\mathrm{ID}_{i}}$ means multiplying all the elements in $\hat{K}_{\mathrm{ID}_{i}}$ by the scalar $r_{\mathrm{ID}_{i}}^{\prime}$. Below we call $\hat{K}_{\mathrm{ID}_{i}}$ the singleton key corresponding to $\mathrm{ID}_{i}$, and $r_{\mathrm{ID}_{i}}^{\prime} \hat{K}_{\mathrm{ID}_{i}}$ is the shifted singleton key for $\mathrm{ID}_{i}$. Note that for the special case $m=1$, we have $r_{\mathrm{ID}}^{\prime}=1$, so KeyGen* degenerates to the original KeyGen.

Extended Decryption. The extended decryption procedure Decrypt ${ }^{*}$ is given a ciphertext $(F, \hat{c})$ together with a set of identities $S=\left\{\mathrm{ID}_{1}, \ldots, \mathrm{ID}_{m}\right\}(m \leq$ $\ell)$ and a matching decryption key $\hat{K}_{S}$. It parses the decryption key as $\hat{K}_{S}=$ $\left[\hat{K}_{\mathrm{ID}_{1}}^{\prime}, \ldots, \hat{K}_{\mathrm{ID}_{m}}^{\prime}\right]$ where the $\hat{K}_{\mathrm{ID}_{i}}^{\prime}$ 's are shifted singleton keys. Namely we have $\hat{K}_{\mathrm{ID}_{i}}^{\prime}=r_{\mathrm{ID}_{i}}^{\prime} \hat{K}_{\mathrm{ID}_{i}}$ where the $\hat{K}_{\mathrm{ID}_{i}}$ 's are singleton keys and $\sum_{i} r_{\mathrm{ID}_{i}}^{\prime}=1(\bmod q)$. Then we use each shifted singleton key to produce $\hat{\boldsymbol{d}}_{\mathrm{ID}_{i}}$ just as in Eq. (5), sets $\hat{\boldsymbol{d}}_{S}=\sum_{i} \hat{\boldsymbol{d}}^{\prime} \mathrm{ID}_{i}$, and recover $\tilde{k}=\left\langle\hat{\boldsymbol{c}}, \hat{\boldsymbol{d}}_{S}\right\rangle$.

Correctness holds since the decryption process in linear: Denote by $\hat{\boldsymbol{d}}_{\mathrm{ID}_{i}}$ the vector that would have been obtained from the singleton key $\hat{K}_{\mathrm{ID}_{i}}$ using Eq. (5)). Then on one hand decryption is linear so we have $\hat{\boldsymbol{d}}_{\mathrm{ID}_{i}}^{\prime}=r_{\mathrm{ID}_{i}}^{\prime} \hat{\boldsymbol{d}}_{\mathrm{ID}_{i}}^{\prime}$. On the other hand by correctness of the basic decryption procedure we know that $\left\langle\hat{\boldsymbol{c}}, \hat{\boldsymbol{d}}_{\mid \mathrm{D}_{i}}\right\rangle=$ $\tilde{k}$. We therefore get

$$
\left\langle\hat{\boldsymbol{c}}, \hat{\boldsymbol{d}}_{S}\right\rangle=\sum_{i}\left\langle\hat{\boldsymbol{c}}, \hat{\boldsymbol{d}}_{\mathrm{ID}_{i}}^{\prime}\right\rangle=\sum_{i}\left\langle\hat{\boldsymbol{c}}, r_{\mathrm{ID}_{i}}^{\prime} \hat{\boldsymbol{d}}_{\mathrm{ID}_{i}}\right\rangle=\sum_{i} r_{\mathrm{ID}_{i}}^{\prime}\left\langle\hat{\boldsymbol{c}}, \hat{\boldsymbol{d}}_{\mathrm{ID}_{i}}\right\rangle=\sum_{i} r_{\mathrm{ID}_{i}}^{\prime} \tilde{k}=\tilde{k}
$$

Key Derivation. Key-derivation uses Boyen's idea of reciprocal keys [8]. Namely, given the public key and any two identities $\mathrm{ID}_{1}$ and $\mathrm{ID}_{2}$, anyone can compute a pair of shifted singleton keys $\delta \hat{K}_{\mathrm{ID}_{1}}$ and $\delta \hat{K}_{\mathrm{ID}_{2}}$ for the same (unknown) scalar factor $\delta$. The procedure for generating these reciprocal keys (which is used as a subroutine for key derivation) is as follows:

ReciprocalKeys $\left(P K, I_{1}, I_{2}\right)$ : Recall that the public key $P K$ depends on the unknown scalars $a, b, s$ (among other things).

- Choose at random $z \in Z_{q}$. The shifted singleton keys $\delta \hat{K}_{\mathrm{ID}_{i}}$ will have $\delta=$ $b z\left(a-\mathrm{ID}_{1}\right)\left(a-\mathrm{ID}_{2}\right)$.

- Choose at random $r_{1}, r_{2} \in Z_{q}$ (which will play the role of $r_{\mathrm{ID}_{1}}$ and $r_{\mathrm{ID}_{2}}$ in the reciprocal keys). 
- Compute $\delta \hat{K}_{\mathrm{ID}_{1}}$ as

$$
\begin{aligned}
& \delta \cdot \frac{s-r_{1}}{a-\mathrm{ID}_{1}} \hat{B}_{1}=\left(a b s-b s \mathrm{ID}_{2}-a b r_{1}+b r_{1} \mathrm{ID}_{2}\right) z \hat{B}_{1} \\
& \delta \cdot r_{1}\left(\boldsymbol{a}_{\ell-2} \times \hat{B}_{1}\right)=\left\{b r_{1} z\left(a^{i+2}-a^{i+1}\left(\mathrm{ID}_{1}+\mathrm{ID}_{2}\right)+a^{i} \mathrm{ID}_{1} \mathrm{ID}_{2}\right) \hat{B}_{1}\right. \\
&: i=0, \ldots, \ell-2\} \\
& \delta \cdot\left(\boldsymbol{a}_{\ell-2} \times \hat{B}_{2}\right)=\left\{b z\left(a^{i+2}-a^{i+1}\left(\mathrm{ID}_{1}+\mathrm{ID}_{2}\right)+a^{i} \mathrm{ID}_{1} \mathrm{ID}_{2}\right) \hat{B}_{2}\right. \\
&: i=0, \ldots, \ell-2\} \\
& \delta \cdot r_{1}\left(\boldsymbol{a}_{\ell-2} \times \hat{B}_{3}\right)=\left\{b r_{1} z\left(a^{i+2}-a^{i+1}\left(\mathrm{ID}_{1}+\mathrm{ID}_{2}\right)+a^{i} \mathrm{ID}_{1} \mathrm{ID}_{2}\right) \hat{B}_{3}\right. \\
&: i=0, \ldots, \ell-2\} \\
& \delta\left(r_{1} a^{\ell-1} \hat{B}_{3}+\operatorname{span}\left(\hat{B}_{5,6}\right)\right)=b r_{1} z\left(a^{\ell+1}-a^{\ell}\left(\mathrm{ID}_{1}+\mathrm{ID}_{2}\right)+a^{\ell-1} \mathrm{ID}_{1} \mathrm{ID}_{2}\right) \hat{B}_{3} \\
&+\operatorname{span}\left(\hat{B}_{5,6}\right)
\end{aligned}
$$

and similarly for $\delta \hat{K}_{\mathrm{ID}_{2}}$ (using $r_{2}$ instead of $r_{1}$ and swapping the roles of $\left.\mathrm{ID}_{1}, \mathrm{ID}_{2}\right)$. Notice that the terms $a^{i} b \hat{B}_{j}$ for $i \in[0, \ell], j=1,2,3$, as well as $b s \hat{B}_{1}, a b s \hat{B}_{1}, a^{\ell+1} b \hat{B}_{3}$, and $\hat{B}_{5,6}$, are all part of the $P K_{3}$ component of the public key.

From the description above it is clear that when $\mathrm{ID}_{1}, \mathrm{ID}_{2} \neq a$, then ReciprocalKeys indeed returns the correct distribution, namely two shifted singleton keys $\delta \hat{K}_{\mathrm{ID}_{1}}, \delta \hat{K}_{\mathrm{ID}_{2}}$ where each $\hat{K}_{\mathrm{ID}}$ is drawn from the same distribution as the singleton keys for ID in KeyGen and $\delta$ is chosen at random in $Z_{q}$ (and independently of $\left.\hat{K}_{\mathrm{ID}_{1}}, \hat{K}_{\mathrm{ID}_{2}}\right)$.

$\operatorname{KeyDerive}\left(P K, S, \hat{K}_{S}, S^{\prime}\right)$ (where $S^{\prime}=\left\{\mathrm{ID}_{1}, \ldots, \mathrm{ID}_{m}\right\}$ and $S \subseteq S^{\prime}$ ). Assume (w.l.o.g.) that $S$ consists of the first $n$ identities in $S^{\prime}$, namely $S=\left\{\mathrm{ID}_{1}, \ldots, \mathrm{ID}_{n}\right\}$ with $n \leq m$. Denote $\hat{K}_{S}=\left\{\hat{K}_{\mathrm{ID}_{1}}^{\prime}, \ldots, \hat{K}_{\mathrm{ID}_{n}}^{\prime}\right\}$, where $\hat{K}_{\mathrm{ID}_{i}}^{\prime}$ is the shifted singleton key for $\mathrm{ID}_{i}$ (consisting of $3 \ell-37$-dimensional vectors of source-group elements).

For $i=1, \ldots, m$, run the ReciprocalKeys procedure from above with identities $\mathrm{ID}_{i}$ and $\mathrm{ID}_{i+1}$ (indexing $\bmod m$ ) to get two shifted singleton keys for these ID's, which we denote by $\hat{L}_{\mathrm{ID}_{i}}, \hat{M}_{\mathrm{ID}_{i+1}}$, respectively. Namely, set

$$
\left(\hat{L}_{\mathrm{ID}_{i}}, \hat{M}_{\mathrm{ID}_{i+1}}\right) \leftarrow \text { ReciprocalKeys }\left(P K, \mathrm{ID}_{i}, \mathrm{ID}_{i+1}\right)
$$

Then for $i \in[1, n]$ set $\hat{K}_{\mathrm{ID}_{i}}^{*}=\hat{K}_{\mathrm{ID}_{i}}^{\prime}+\hat{L}_{\mathrm{ID}_{i}}-\hat{M}_{\mathrm{ID}_{i}}$, and for $i \in[n+1, m]$ set $\hat{K}_{\mathrm{ID}_{i}}^{*}=\hat{L}_{\mathrm{ID}_{i}}-\hat{M}_{\mathrm{ID}_{i}}$ (where addition and subtraction is element-wise). The new key is $\hat{K}_{S}=\left[\hat{K}_{\mathrm{ID}_{1}}^{*}, \ldots, \hat{K}_{\mathrm{ID}_{m}}^{*}\right]$. In Lemma 2] below we show that this KeyDerive procedure induces almost the same distribution as KeyGen over the decryption key $\hat{K}_{S^{\prime}}$.

Lemma 2. For every $S \subseteq S^{\prime}$ (with $\left|S^{\prime}\right|=m$ ) and every secret key $\hat{K}_{S}$ corresponding to $S$, the procedure KeyDerivation $\left(P K, S, \hat{K}_{S}, S^{\prime}\right)$ draws from a distribution at most $O(m / q)$ away from that of $\operatorname{KeyGen}\left(P K, S K, S^{\prime}\right)$.

Proof. Observe that every 7 -vector in a singleton key $\hat{K}_{\text {ID }}$ corresponding to identity ID (as computed by KeyGen) is of the form

$$
\left(\operatorname{expr}(a, s, \mathrm{ID})+r_{\mathrm{ID}} \operatorname{expr}^{\prime}(a, s, \mathrm{ID})\right) \cdot \hat{B}_{k}
$$


where $r_{\mathrm{ID}}$ is the scalar that was chosen for this singleton key, $\hat{B}_{k}$ is one specific row of the matrix $\hat{B}$, and $\operatorname{expr}(a, s, \mathrm{ID}), \operatorname{expr}^{\prime}(a, s, \mathrm{ID})$ are two fixed scalar-valued expressions that depend only on the scalars $a, s$ from the master secret key and on the identity ID. (Note that either $\operatorname{expr}\left(a, s\right.$, ID) or $\operatorname{expr}^{\prime}(a, s$, ID) can be zero, but not both.)

Considering the same vector in all the shifted singleton keys in $\hat{K}_{S}$, we have a collection of $n$ vectors, $\hat{\boldsymbol{x}}_{1}, \ldots, \hat{\boldsymbol{x}}_{n}$, where $\hat{\boldsymbol{x}}_{i}=r_{\mathrm{ID}_{i}}^{\prime}\left(\operatorname{expr}\left(a, s, \mathrm{ID}_{i}\right)+r_{\mathrm{ID}_{i}} \operatorname{expr}(a, s\right.$, $\left.\left.\mathrm{ID}_{i}\right)\right) \cdot \hat{B}_{k}$, and the scalars $r_{\mathrm{ID}_{i}}^{\prime}$ satisfy $\sum_{i} r_{\mathrm{ID}_{i}}^{\prime}=1$. For notational convenience, for $i \in[n+1, m]$ we denote $r_{\mathrm{ID}_{i}}=r_{\mathrm{ID}_{i}}^{\prime}=0$ and $\hat{\boldsymbol{x}}_{i}=\hat{\mathbf{0}}$ (so we still have $\hat{x}_{i}$ 's of the right format with $\sum_{i} r_{\mathrm{ID}_{i}}^{\prime}=1$, even when we consider all $m$ elements). Similarly considering the same vector in all the shifted singleton keys that are generated by ReciprocalKeys, we have vectors $\hat{\boldsymbol{y}}_{1} \ldots \hat{\boldsymbol{y}}_{m}$ (from the $\hat{L}_{\mathrm{ID}_{i}}$ 's) and $\hat{\boldsymbol{z}}_{1} \ldots \hat{\boldsymbol{z}}_{m}$ (from the $\hat{M}_{\mathrm{ID}_{i}}$ 's) of the form

$$
\begin{aligned}
\hat{\boldsymbol{y}}_{i} & =\delta_{i}\left(\operatorname{expr}\left(a, s, \mathrm{ID}_{i}\right)+\rho_{i} \operatorname{expr}^{\prime}\left(a, s, \mathrm{ID}_{i}\right)\right) \cdot \hat{B}_{k} \\
\text { and } \hat{\boldsymbol{z}}_{i} & =\delta_{i-1}\left(\operatorname{expr}\left(a, s, \mathrm{ID}_{i}\right)+\tau_{i} \operatorname{expr}^{\prime}\left(a, s, \mathrm{ID}_{i}\right)\right) \cdot \hat{B}_{k}
\end{aligned}
$$

where all the scalars $\delta_{i}, \rho_{i}, \tau_{i}, i=1 \ldots m$, are chosen at random in $Z_{q}$ (and indexing is $\bmod m$, so $\left.\delta_{0}=\delta_{m}\right)$. Hence the corresponding element in the shifted singleton key $\hat{K}_{\mathrm{ID}_{i}}^{*}$ is

$\hat{\boldsymbol{x}}_{i}+\hat{\boldsymbol{y}}_{i}-\hat{\boldsymbol{z}}_{i}=\left(\left(r_{\mathrm{ID}_{i}}^{\prime}+\delta_{i}-\delta_{i-1}\right) \operatorname{expr}\left(a, s, \mathrm{ID}_{i}\right)+\left(r_{\mathrm{ID}_{i}}^{\prime} r_{\mathrm{ID}_{i}}+\delta_{i} \rho_{i}-\delta_{i-1} \tau_{i}\right) \operatorname{expr}\left(a, s, \mathrm{ID}_{i}\right)\right) \hat{B}_{k}$

Assuming that $r_{\mathrm{ID}_{i}}^{\prime}+\delta_{i}-\delta_{i-1} \neq 0$, we can denote

$$
r_{\mathrm{ID}_{i}}^{* *} \stackrel{\text { def }}{=} r_{\mathrm{ID}_{i}}^{\prime}+\delta_{i}-\delta_{i-1} \text { and } r_{\mathrm{ID}_{i}}^{*} \stackrel{\text { def }}{=} \frac{r_{\mathrm{ID}_{i}}^{\prime} r_{\mathrm{ID}_{i}}+\delta_{i} \rho_{i}-\delta_{i-1} \tau_{i}}{r_{\mathrm{ID}_{i}}^{\prime}+\delta_{i}-\delta_{i-1}}
$$

and then we have $\hat{\boldsymbol{x}}_{i}+\hat{\boldsymbol{y}}_{i}-\hat{\boldsymbol{z}}_{i}=r_{\mathrm{ID}_{i}}^{* *}\left(\operatorname{expr}\left(a, s, \mathrm{ID}_{i}\right)+r_{\mathrm{ID}_{i}}^{*} \operatorname{expr}^{\prime}\left(a, s, \mathrm{ID}_{i}\right)\right) \hat{B}_{k}$, which is of the right form, and indeed the scalars $r_{\mathrm{ID}_{i}}^{* *}$ satisfy

$$
\sum_{i=1}^{m} r_{\mathrm{ID}_{i}}^{* *}=\sum_{i=1}^{m} r_{\mathrm{ID}_{i}}^{\prime}+\sum_{i=1}^{m} \delta_{i}-\sum_{i=1}^{m} \delta_{i-1}=\sum_{i=1}^{m} r_{\mathrm{ID}_{i}}^{\prime}=1
$$

Since the $\delta_{i}$ 's are random and independent then the $r_{\mathrm{ID}_{i}}^{* *}$ 's are also random and independent subject to the constraint that their sum is one. Finally, assuming that none of the $r_{\mathrm{ID}_{i}}^{* *}$ 's is zero and also none of the $\delta_{i}$ 's are zero (which happens with probability at least $1-O(\mathrm{~m} / q)$ ) then all the $r_{\mathrm{ID}_{i}}^{*}$ 's are random and independent (since the $\tau_{i}$ 's and $\rho_{i}$ 's are).

\section{Security of Our System}

Theorem 2. The IBBE system from Section 5 is secure under the BDHESet assumption and the decision Linear assumption. Specifically, for an $\ell$-depth 
hierarchy, groups $\mathbb{G}, \mathbb{G}_{T}$ of order $q$, and an adversary that makes upto $q^{*}$ keyextraction queries, we have $\operatorname{AdvIBBE}^{\mathcal{E}}(\log q, \ell) \leq \operatorname{AdvBDHE}\left(\mathbb{G}, \mathbb{G}_{T}\right)+\operatorname{AdvLinear}$ $\left(\mathbb{G}, \mathbb{G}_{T}\right)$, where the BDHE-Set instances are of size $O\left(\ell+q^{*}\right)$.

The proof is found in the long version 14. On a very high level, the proof consists of four games: Game 0 is the actual interaction of the adversary with our system, in Game 1 we use decryption rather than encryption to compute the KEM key corresponding to the challenge ciphertext (which has no effect on the outcome), in Game 2 we add a component of $\hat{B}_{7}$ to the secret keys (which is indistinguishable by the Linear assumption), and in Game 3 we add a component of $\hat{A}_{7}$ to the challenge ciphertext vector (thus making the KEM key statistically independent of the adversary's view).

The main reduction then proves indistinguishability of Game 3 from Game 2 based on the BDHE-Set assumption. That reduction follows the hash-proof approach: The simulator generates the challenge ciphertext so that this is either a valid ciphertext or an invalid one, depending on whether the input of the simulator is a YES instance or a NO instance of the decision BDHE-Set problem. In our case, a valid ciphertext is spanned by the rows $\hat{A}_{1,2,3,4}$, and an invalid ciphertext also has a component of $\hat{A}_{7}$. The secret keys have a random $\hat{B}_{7}$ component in them, so an invalid ciphertext is decrypted to a random KEM key (while a valid ciphertext are always decrypted to the "right KEM key").

In the reduction itself, the simulator gets as input source-group elements $\hat{a}^{i}=$ $a^{i} \cdot \hat{1}$ for all $i \in \mathcal{S}$ and two additional source-group elements $\hat{z}_{1}, \hat{z}_{2}$, and uses these elements to answer all the queries of the adversary: Very roughly, it chooses a random polynomial $\mathrm{H}(x)$ of high-enough degree over $Z_{q}$, sets $s=\mathrm{H}(a)$ for the master secret key, $r_{\mathrm{ID}}=H(\mathrm{ID})$ in all the key-reveal queries, and $\mathrm{F}=\mathrm{H} \bmod \mathrm{P}$ for the challenge ciphertext. To compute the appropriate terms, the simulator uses the powers $\hat{a^{i}}$ from its input. The main challenge is to make the set $\mathcal{S}$ "large enough" so the simulator can produce the entire view of the adversary from the elements $\hat{a}^{i}$ that it knows, while at the same time ensuring that $\mathcal{S}$ is "small enough" so that the target integer $m$ is not in $\mathcal{S}+\mathcal{S}$ (since otherwise the problem becomes easy).

\section{References}

1. Boneh, D., Boyen, X.: Efficient Selective-ID Secure Identity Based Encryption Without Random Oracles. In: Cachin, C., Camenisch, J.L. (eds.) EUROCRYPT 2004. LNCS, vol. 3027, pp. 223-238. Springer, Heidelberg (2004)

2. Boneh, D., Boyen, X.: Secure Identity Based Encryption Without Random Oracles. In: Franklin, M. (ed.) CRYPTO 2004. LNCS, vol. 3152, pp. 443-459. Springer, Heidelberg (2004)

3. Boneh, D., Boyen, X., Goh, E.-J.: Hierarchical Identity Based Encryption with Constant Size Ciphertexts. In: Cramer, R. (ed.) EUROCRYPT 2005. LNCS, vol. 3494, pp. 440-456. Springer, Heidelberg (2005)

4. Boneh, D., Boyen, X., Shacham, H.: Short group signatures. In: Franklin, M. (ed.) CRYPTO 2004. LNCS, vol. 3152, pp. 41-55. Springer, Heidelberg (2004) 
5. Boneh, D., Franklin, M.: Identity Based Encryption from the Weil Pairing. In: Kilian, J. (ed.) CRYPTO 2001. LNCS, vol. 2139, pp. 213-229. Springer, Heidelberg (2001)

6. Boneh, D., Gentry, C., Hamburg, M.: Space Efficient Identity Based Encryption without Pairings. In: Proceedings of FOCS 2007, pp. 647-657. IEEE, Los Alamitos (2007)

7. Boneh, D., Gentry, C., Waters, B.: Collusion Resistant Broadcast Encryption with Short Ciphertexts and Private Keys. In: Shoup, V. (ed.) CRYPTO 2005. LNCS, vol. 3621, pp. 258-275. Springer, Heidelberg (2005)

8. Boyen, X.: General Ad Hoc Encryption from Exponent Inversion IBE. In: Naor, M. (ed.) EUROCRYPT 2007. LNCS, vol. 4515, pp. 394-411. Springer, Heidelberg (2007)

9. Canetti, R., Halevi, S., Katz, J.: A Forward-Secure Public-Key Encryption Scheme. In: Biham, E. (ed.) EUROCRYPT 2003. LNCS, vol. 2656, pp. 255-271. Springer, Heidelberg (2003)

10. Canetti, R., Halevi, S., Katz, J.: Chosen-Ciphertext Security from Identity-Based Encryption. In: Cachin, C., Camenisch, J.L. (eds.) EUROCRYPT 2004. LNCS, vol. 3027, pp. 207-222. Springer, Heidelberg (2004)

11. Cocks, C.: An Identity Based Encryption Scheme Based on Quadratic Residues. In: IMA Int. Conf. 2001 (2001)

12. Cramer, R., Shoup, V.: Universal Hash Proofs and a Paradigm for Adaptive Chosen Ciphertext Secure Public-Key Encryption. In: Knudsen, L.R. (ed.) EUROCRYPT 2002. LNCS, vol. 2332, pp. 45-64. Springer, Heidelberg (2002)

13. Gentry, C.: Practical Identity-Based Encryption without Random Oracles. In: Vaudenay, S. (ed.) EUROCRYPT 2006. LNCS, vol. 4004, pp. 445-464. Springer, Heidelberg (2006)

14. Gentry, C., Halevi, S.: Hierarchical Identity Based Encryption with Polynomially Many Levels, http://eprint.iacr.org/2008/383

15. Gentry, C., Silverberg, A.: Hierarchical ID-Based Cryptography. In: Zheng, Y. (ed.) ASIACRYPT 2002. LNCS, vol. 2501, pp. 548-566. Springer, Heidelberg (2002)

16. Gentry, C., Waters, B.: Adaptive Security in Broadcast Encryption Systems (manuscript, 2008), http://eprint.iacr.org/2008/268

17. Horwitz, J., Lynn, B.: Toward Hierarchical Identity-Based Encryption. In: Knudsen, L.R. (ed.) EUROCRYPT 2002. LNCS, vol. 2332, pp. 466-481. Springer, Heidelberg (2002)

18. Maurer, U.M., Yacobi, Y.: Non-interative Public-Key Cryptography. In: Davies, D.W. (ed.) EUROCRYPT 1991. LNCS, vol. 547, pp. 498-507. Springer, Heidelberg (1991)

19. Shamir, A.: Identity-Based Cryptosystems and Signature Schemes. In: Blakely, G.R., Chaum, D. (eds.) CRYPTO 1984. LNCS, vol. 196, pp. 47-53. Springer, Heidelberg (1985)

20. Shi, E., Waters, B.: Delegating Capabilities in Predicate Encryption Systems. In: Aceto, L., Damgård, I., Goldberg, L.A., Halldórsson, M.M., Ingólfsdóttir, A., Walukiewicz, I. (eds.) ICALP 2008, Part II. LNCS, vol. 5126, pp. 560-578. Springer, Heidelberg (2008)

21. Weisstein, E.W.: Sylvester Matrix. From MathWorld, a Wolfram Web Resource, http://mathworld.wolfram.com/SylvesterMatrix.html

22. Waters, B.: Efficient Identity Based Encryption without Random Oracles. In: Cramer, R. (ed.) EUROCRYPT 2005. LNCS, vol. 3494, pp. 114-127. Springer, Heidelberg (2005) 